\title{
BMJ Open General practitioner and pharmacist collaboration: does this improve risk factors for cardiovascular disease and diabetes? A systematic review protocol
}

Kanika Chaudhri, ${ }^{\oplus 1,2}$ Adina Hayek, ${ }^{3}$ Hueiming Liu, ${ }^{\circledR 2,4}$ Rohina Joshi ${ }^{\odot, 5,6,7}$

To cite: Chaudhri K, Hayek A, Liu H, et al. General practitioner and pharmacist collaboration: does this improve risk factors for cardiovascular disease and diabetes? A systematic review protocol. BMJ Open 2019;9:e027634. doi:10.1136/ bmjopen-2018-027634

- Prepublication history and additional material for this paper are available online. To view please visit the journal (http:// dx.doi.org/10.1136/bmjopen2018-027634).

Received 1 November 2018

Revised 25 June 2019

Accepted 1 July 2019

\section{Check for updates}

(C) Author(s) (or their employer(s)) 2019. Re-use permitted under CC BY-NC. No commercial re-use. See rights and permissions. Published by BMJ.

For numbered affiliations see end of article.

Correspondence to Kanika Chaudhri; kchaudhri@georgeinstitute. org.au

\section{ABSTRACT}

Introduction Cardiovascular disease (CVD) remains a major cause of morbidity and premature mortality globally. Despite the availability of low-cost evidence based medicines, there is a significant treatment gap in those with established or at high risk of CVD in the primary care setting. Pharmacist-based interventions have shown to improve patient outcomes for many chronic diseases including CVD. However, there is little synthesised evidence that has examined the effects of collaborative care between general practitioners (GPs) and pharmacists on patients' cardiovascular risk outcomes. This protocol aims to outline the methods employed in a systematic review of current literature to assess whether interprofessional collaboration between GPs and pharmacists has an impact on improving cardiovascular risk outcomes among patients in the primary care setting.

Methods and analysis Randomised controlled trials (RCTs) will be identified through database searches, scanning reference lists of relevant studies, hand searching of key journals and citation searching of key papers. Two independent reviewers will screen studies against eligibility criteria and extract data using standardised forms. Databases including MEDLINE, EMBASE, Cochrane, CINAHL and International Pharmaceutical Abstracts, will be searched from the beginning of each database until October 2018. Primary outcome includes improvement in cardiovascular risk factors, such as hypertension, due to GP and pharmacist cooperation. Secondary outcome is to describe the different types of GP and pharmacist collaborative models of care. A narrative synthesis of findings will be presented. A meta-analysis will be performed if the data are homogenous.

Ethics and dissemination This study does not require ethics approval. The results of the systematic review described within this protocol will be disseminated through presentations at relevant conferences and publication in a peer-reviewed journal. The methods will be used to inform future reviews.

PROSPERO registration number CRD42017055259.

\section{BACKGROUND}

Cardiovascular disease (CVD) continues to be a major contributor to mortality, morbidity and health expenditure in higher income

\section{Strengths and limitations of this study}

- The systematic review described within this protocol will provide a synthesis of randomised controlled trials, outlining the relationship between interprofessional collaboration between general practitioner (GP) and pharmacist collaboration and its impact on patient's cardiovascular risk in the primary care setting.

- Scanning reference lists of relevant studies, hand searching of key journals and citation searching of key papers will identify articles additional articles that may be overlooked by the search strategy.

- Meta-analysis will be performed on data if variables are comparable and there is sufficient data.

- The review described will be limited to GPs and pharmacists. We acknowledge that collaborative care includes other allied professionals such as nurse practitioners, dietitians, physiotherapists, who will not be included in the search criteria.

- The review outlined in this protocol will measure disease-centred outcomes rather than patient-centred outcomes.

countries and globally. ${ }^{12}$ CVD is the second highest cause of years of life lost to premature mortality in most high income nations. ${ }^{3}$ Low-income and middle-income nations also have a rising prevalence of chronic disease. ${ }^{45}$ They have a proportionately younger population, yet the age-standardised mortality rate for CVD is greater than that of higher income nations. ${ }^{4}$ This global trend in CVD indicates the need for transition of health research and health services to models of care, which are patient centred and improve health outcomes.

Despite the availability of low-cost evidence based treatment for CVD, there still remains is a significant treatment gap in those with established or at high risk of CVD in the primary care setting. ${ }^{6} 7$ This gap in the uptake of preventative measures outlines an area to focus current resources with general 
practice being the ideal setting due to frequency of patient encounters. For example, in high income countries like Australia, $83 \%$ of Australians attend a general practitioner (GP) every year. ${ }^{8}$ These consultations are opportunities to implement primary and secondary prevention measures including lifestyle advice, smoking cessation/avoidance advice and guideline-based pharmacotherapy. ${ }^{9}$ However, with the rise of chronic disease, misdistribution of physicians across urban and rural regions and the high costs involved in physician-centred models of care, new models have been suggested including the utilisation of the skills of other health professionals such as pharmacists. ${ }^{10}$

Pharmacist-based interventions alone have been effective in the improvement of CVD outcomes. ${ }^{11}{ }^{12}$ Studies have shown that interventions involving pharmacists have been effective to reduce $\mathrm{HbAlC}$ in diabetic patients, ${ }^{13}$ improve systolic blood pressure control ${ }^{13}$ and reduce total cholesterol $^{1415}$ measurements. In spite of this evidence, pharmacists remain an underused member of the multidisciplinary healthcare team within primary care. ${ }^{16}$

Despite the growing need to evaluate the use of multidisciplinary team models, ${ }^{17}$ especially in chronic disease management. ${ }^{18}$ There is little synthesised evidence available that analyses the effect of collaboration between GP and pharmacists on CVD outcomes. Two published systematic reviews have investigated the effect of the interprofessional relationship between GPs and pharmacists on patient outcomes. The first review, written in French incorporated literature up until December 2011. ${ }^{19}$ This review was not disease or outcome specific. The second systematic review aimed to measure the impact of medication review on patient behaviour and did not evaluate the effect of the collaboration on patient outcomes. ${ }^{20}$ Three other systematic reviews assessed pharmacy interventions in the primary care setting, but did not focus on collaborative care between GPs and pharmacists. ${ }^{21-23}$ This emphasises the lack of current evidence in the growing area of GP and pharmacist collaboration.

Therefore, this protocol aims to outline the methods that will be used in a systematic review of the current literature to assess whether GP and pharmacist collaboration improves patients' risk factors or CVD management within the primary care setting.

\section{METHODS AND ANALYSIS}

This systematic review focuses on randomised control trials (RCTs) of interprofessional collaborations between GPs and pharmacists, assessing a reduction of patient cardiovascular risk or improvement in CVD management in the primary care setting. We have described our methods as per Preferred Reporting Items for Systematic Review and Meta-Analysis (PRISMA) for protocol recommendations, and this checklist is included as online supplementary additional file 1 . Final reporting of this study will be compliant with the main PRISMA statement.

\section{Patients and public involvement}

Patients and public were not involved in writing this protocol.

\section{Eligibility criteria}

Definitions as per PICO-D have been adapted for the purpose of this review:

Participants-participants include any patients at risk of CVD or who have established CVD or associated conditions. Definitions for CVD and related conditions are based on the Australian Institute for Health and Welfare publication. ${ }^{1}$ CVD and related conditions include: coronary disease, stroke, heart failure, cardiomyopathies, rheumatic fever, rheumatic heart disease, peripheral vascular disease, congenital heart defects, type 2 diabetes mellitus and chronic renal insufficiency (excluding rheumatic fevers, rheumatic heart disease and congenital heart defects based on different aetiology/risk factors and management). For the purpose of this review, rheumatic fever, rheumatic heart disease and congenital heart defects will be excluded from the definition due to different disease aetiology and prevention/management strategies. Risk factors for these conditions include tobacco smoking, sedentary lifestyle, poor nutritional status, excessive alcohol consumption, hypertension, hyperlipidaemia, overweight and obesity and depression.

Interventions-interventions include the effect of collaboration between GPs and pharmacists versus usual care in the primary care setting. For the purpose of this systematic review, collaboration between GPs and pharmacists will include two-way collaboration for the benefit of the patient. Two-way collaboration is described as back and forth communication between the GP and pharmacist. This can be verbal (face to face or phone call) or written (letters, fax, email or medical note) communication. One-way communication will be excluded, as it does not meet our papers criteria of true collaboration. Both GPs and pharmacists must both practice in the primary care setting.

Comparator-comparator is usual care.

Outcomes-a change in patients' CVD risk, CVD parameters or associated conditions (diabetes mellitus, chronic kidney disease) parameters. Expected quantitative cardiovascular outcomes included blood pressure, total cholesterol (low-density lipoproteins/high-density lipoproteins/triglyceride changes), HbA1C, weight and smoking.

Timing-years of search from beginning of database.

Design-RCTs will be considered eligible for this review.

Exclusion criteria-articles will be excluded if they are not a journal article, not a report based on empirical research (eg, protocol, editorial), reviews and not human research.

\section{Information sources}

This systematic review will involve a search of MEDLINE, EMBASE, Cochrane, CINAHL and International Pharmaceutical Abstracts databases. Further studies will also 
be obtained from scanning reference lists of relevant studies, hand-searching of key journals (including Circulation, Pharmacotherapy, Clinical Therapeutics, Journal of Clinical Pharmacy and Therapeutics, Journal of the American Pharmacists Association, and Pharmacy Practice) and citation searching of key papers identified for inclusion. Any study published prior to October 2018 will be included in the review.

\section{Search strategy}

A search strategy was developed with the initial support of a medical research librarian (online supplementary additional file 2). Keywords included pharmacists, physicians and randomised controlled trials.

\section{Study records}

\section{Data management}

After searching, the shortlisted articles will be exported to Endnote X9 (Thomson Reuters, New York, USA) for storage of study records, abstracts and full text articles. Data will be stored on a password protected server-based platform, that accessible by both reviewers. At each stage of the data selection process (eg, after consolidation of all articles prior to assessing eligibility based on title and abstract), back up files of the Endnote database will be made in order to retrace any steps, as needed in the review process.

\section{Selection process}

The process of study selection will be conducted by two researchers. In the initial screening stage, the authors will conduct a title search and identify abstracts, which potentially meet the criteria for study selection. Abstracts for which it is unclear whether to include in the study will be further assessed against the criteria after acquiring full text articles. This will be done independently to reduce risk of bias. Discrepancies between two reviews will be resolved by consultation with the senior author. Detailed notations of decisions made to include or exclude studies and the rationale for these decisions will be documented. The flow of studies through selection process, together with reasons for exclusion at the full-text screen will be reported using a modified PRIMSA diagram.

Difficulties may arise when assessing studies for inclusion, and therefore rules have been established to clarify the process that will be used in this study. One common cause of confusion occurs when there is a lack of information provided in the report of a study. Where the final report does not include sufficient detail, the published protocol will be reviewed to extract further methodological details. Where a single study has multiple identified papers (duplicate reports), the paper with the maximum information will be included with all other reports from the study being referenced.

\section{Data collection process}

Once the studies for inclusion have been identified, information outlined in the standardised data extraction form (online supplementary additional file 3 ) will be collected.
Data from all included studies will be extracted. The form will be piloted and optimised by the two reviewers using a subset of three randomly selected studies that satisfy the eligibility criteria. The two reviews will independently extract data from the rest of the included list of articles. Study authors will be emailed where there is a lack of information or need for clarification in the report of a study.

\section{Data items}

The following data will be extracted from the included studies:

1. General study information including study title and citation, study authors, year of publication, publication journal and any additional published reports.

2. Characteristics of the study including aim or objectives of the study, target condition or risk factor studied, study design and criteria for recruitment of GPs/pharmacists/patients.

3. Participant characteristics including numbers of patients, pharmacists and GPs in the control and intervention groups, baseline demographics and dropout rates.

4. Intervention characteristics including location of study, duration of the intervention, description of the intervention, length between and number of follow-ups, methods of GP/pharmacist collaboration and any coexisting interventions implemented.

5. Outcomes result of primary outcome and statistical significance, statistical methods used documentation of specific quantitative and qualitative outcomes of interest, Cochrane collaboration risk of bias assessment of RCTs, any economic evaluation and overall study conclusion.

\section{Outcomes and prioritisation}

The primary outcome of this study is to assess the effects of GP and pharmacist cooperation on patients' risk factor management for CVD and associated conditions (including diabetes mellitus and chronic kidney disease). The secondary outcome is to describe the different types of GP and pharmacist cooperation models.

These outcomes will be relevant to current clinical models that encourage interdisciplinary communication and increasing role of the community pharmacists in chronic disease management. This review will provide evidence of the effectiveness of non-dispensing pharmacists co-located at general practice.

\section{Risk of bias in individual studies}

The risk of bias will be ascertained by two reviewers in independently using The Cochrane Risk of Bias Tool. ${ }^{24}$ The assessment will be performed at study level and will focus on selection, performance, detection, attrition and reporting bias. Additionally, Grades of Recommendation, Assessment, Development and Evaluation system will be used to summarise the quality of evidence for each outcome. $^{25}$ 


\section{Data synthesis and analysis}

Studies will be included in data synthesis if they fulfil the eligibility criteria. Data will be presented in a descriptive narrative, and supplemented with tables and figures where appropriate. Depending on papers included, there may be a subgroup analysis of further exploration of any differences of the barriers and facilitators to intervention implementation. Meta-analysis will be performed on data if variables are comparable and there is sufficient data.

\section{ETHICS AND DISSEMINATION}

This study does not require ethics approval as it is a systematic review. The results of the review described within this protocol will be disseminated through presentations at relevant conferences and publication within a peer-reviewed journal. The methods employed within this review will be used to inform future reviews, specifically those exploring the impact of GP and pharmacist collaboration.

\section{Author affiliations}

${ }^{1}$ Cardiovascular Division, The George Insitute for Global Health, Newtown, New South Wales, Australia

${ }^{2}$ Faculty of Medicine, Univesity of New South Wales, Sydney, New South Wales, Australia

${ }^{3}$ Health Services Research, The George Institute for Global Health, Newtown, New South Wales, Australia

${ }^{4}$ Health Economics and Process Evaluation, The George Institute for Global Health, Newtown, New South Wales, Australia

${ }^{5}$ Office of the Chief Scientist, The George Institute for Global Heath, Newtown, New South Wales, Australia

${ }^{6}$ The George Institute for Global Health, New Delhi, India

${ }^{7}$ Faculty of Medicine, University of Sydney, Camperdown, New South Wales, Australia

Contributors $\mathrm{AH}$ conducted the scoping searches and designed the data extraction forms. KC updated the search, drafted the manuscript and now manages the overall study. KC and $\mathrm{HL}$ will be involved in study selection, data extraction, synthesis and analysis. Study was conceived by RJ. All authors read and approved the manuscript.

Funding The authors have not declared a specific grant for this research from any funding agency in the public, commercial or not-for-profit sectors.

Competing interests None declared.

Patient consent for publication Not required.

Provenance and peer review Not commissioned; externally peer reviewed.

Open access This is an open access article distributed in accordance with the Creative Commons Attribution Non Commercial (CC BY-NC 4.0) license, which permits others to distribute, remix, adapt, build upon this work non-commercially, and license their derivative works on different terms, provided the original work is properly cited, appropriate credit is given, any changes made indicated, and the use is non-commercial. See: http://creativecommons.org/licenses/by-nc/4.0/.

\section{REFERENCES}

1. Australian Institute of Health and Welfare. Cardiovascular disease: Australian facts. Cardiovascular disease series Cat no CVD 53. Canberra: AlHW, 2011.

2. World Health Organisation. Prevention of cardiovascular disease: guidelines for assessment and management of total cardiovascular risk, 2007.
3. WHO statistical profile. Country statistics and global health estimates by WHO and UN partners. 2015. http://who.int/countries/aus/en/ (Accessed 23 Sep 15).

4. Di Cesare M, Khang Y, Asaria P, et al. Non-Communicable Diseases 3: Inequalities in non-communicable diseases and effective responses. Lancet 2013;381:585-97.

5. Muka T, Imo D, Jaspers L, et al. The global impact of noncommunicable diseases on healthcare spending and national income: a systematic review. Eur J Epidemiol 2015;30:251-77.

6. Webster RJ, Heeley EL, Peiris DP, et al. Gaps in cardiovascular disease risk management in Australian general practice. Med J Aust 2009;191:324-9.

7. Heeley EL, Peiris DP, Patel AA, et al. Cardiovascular risk perception and evidence--practice gaps in Australian general practice (the AusHEART study). Med J Aust 2010;192:254-9.

8. Royal Australian College of General Practitioners. Guidelines for preventive activities in general practice: East Melbourne, 2012.

9. Fulcher GR, Amarena JV, Conner GW, et al. Practical Implementation Taskforce for the Prevention of Cardiovascular Disease. Prevention of cardiovascular disease: an evidence-based clinical aid 2004. Med J Aust 2004;181:F1-16.

10. Glynn LG, Valderas JM, Healy P, et al. The prevalence of multimorbidity in primary care and its effect on health care utilization and cost. Fam Pract 2011;28:516-23

11. McNamara KP, O'Reilly SL, Dunbar JA, et al. A pilot study evaluating multiple risk factor interventions by community pharmacists to prevent cardiovascular disease: the PAART CVD pilot project. Ann Pharmacother 2012;46:183-91.

12. Altowaijri A, Phillips CJ, Fitzsimmons D. A systematic review of the clinical and economic effectiveness of clinical pharmacist intervention in secondary prevention of cardiovascular disease. $J$ Manag Care Pharm 2013;19:408-16.

13. Machado M, Bajcar J, Guzzo GC, et al. Sensitivity of patient outcomes to pharmacist interventions. Part I: systematic review and meta-analysis in diabetes management. Ann Pharmacother 2007;41:1569-82.

14. Machado M, Bajcar J, Guzzo GC, et al. Sensitivity of patient outcomes to pharmacist interventions. Part II: Systematic review and meta-analysis in hypertension management. Ann Pharmacother 2007;41:1770-81.

15. Blenkinsopp A, Anderson C, Armstrong M. Systematic review of the effectiveness of community pharmacy-based interventions to reduce risk behaviours and risk factors for coronary heart disease. J Public Health Med 2003;25:144-53.

16. McMillan SS, Wheeler AJ, Sav A, et al. Community pharmacy in Australia: a health hub destination of the future. Res Social Adm Pharm 2013;9:863-75.

17. Zwarenstein M, Goldman J, Reeves S. Interprofessional collaboration: effects of practice-based interventions on professional practice and healthcare outcomes. Cochrane Database Syst Rev 2009;3:CD000072.

18. Cramm JM, Nieboer AP. In The Netherlands, rich interaction among professionals conducting disease management led to better chronic care. Health Aff 2012;31:2493-500.

19. Michot P, Catala O, Supper I, et al. [Cooperation between general practitioners and pharmacists: a systematic review]. Sante Publique 2013;25:331-41.

20. Kwint HF, Bermingham $L$, Faber $A$, et al. The relationship between the extent of collaboration of general practitioners and pharmacists and the implementation of recommendations arising from medication review: a systematic review. Drugs Aging 2013;30:91-102.

21. Tan EC, Stewart K, Elliott RA, et al. Pharmacist services provided in general practice clinics: a systematic review and meta-analysis. Res Social Adm Pharm 2014;10:608-22.

22. Santschi V, Chiolero A, Burnand B, et al. Impact of pharmacist care in the management of cardiovascular disease risk factors: a systematic review and meta-analysis of randomized trials. Arch Intern Med 2011;171:1441-53.

23. Santschi V, Chiolero A, Colosimo AL, et al. Improving blood pressure control through pharmacist interventions: a meta-analysis of randomized controlled trials. J Am Heart Assoc 2014;3:e000718.

24. Higgins JP, Altman DG, Gøtzsche PC, et al. The Cochrane Collaboration's tool for assessing risk of bias in randomised trials. BMJ 2011;343:d5928.

25. Guyatt G, Oxman AD, Akl EA, et al. GRADE guidelines: 1. Introduction-GRADE evidence profiles and summary of findings tables. 2011;64:383-94. 